\title{
Editorial
}

\section{Apoptosis and autoimmunity: two sides to the coin}

\author{
Mauro Piacentini*,1,2 \\ 1 Department of Biology, University of Rome 'Tor Vergata', Rome, Italy \\ 2 Laboratory of EM and Cell Biology, 'L. Spallanzani' IRCCS, Rome, Italy \\ * corresponding author: M. Piacentini, Laboratory of EM and Cell Biology, \\ 'L. Spallanzani' IRCCS, Rome, Italy
}

Alteration of cell death homeostasis is an essential event characterizing the pathogenesis of many diseases. There is growing consensus on the fact that autoimmunity is a consequence of defects occurring in the signaling, execution and clearance pathways involved in apoptosis. ${ }^{1}$ Extensive post-translational modifications of cellular proteins occur in apoptotic cells, resulting in notable changes not only in the structure of the dying cells, but also in their antigenic properties. ${ }^{2}$ However, the harmful consequences intrinsic of this event are prevented by the rapid clearance of cells undergoing apoptosis by neighboring phagocytes. ${ }^{3}$ As a consequence, any perturbation of the dynamic equilibrium between the generation and clearance of apoptotic cells will expose the immune system to harmful apoptosis-generated autoantigens. In this issue of Cell Death and Differentiation Rosen and Casciola-Rosen ${ }^{4}$ provide an overview of how the impairment of apoptosis, with the consequent exposition of cryptic determinants, might result in pathogenic events leading to the development of systemic autoimmune disorders. In an accompanying review, Ohsako and Elkon ${ }^{5}$ critically describe the molecular events thought to be responsible for the failure of apoptosis signaling pathways, in particular of the TNF receptor family, in organ-specific autoimmune diseases (thyroiditis, multiple sclerosis and insulin-dependent diabetes mellitus).

The first molecular evidence of the potential link between apoptosis and autoimmunity was the demonstration that $I p r$ and gld mice, well known animal models of autoimmune diseases, are loss-of-function mutants of the CD95 and CD95 ligand genes respectively. ${ }^{6}$ The relevance of these findings was recently confirmed by the fact that patients with autoimmune lymphoproliferative disorders have also been found to bear mutations of the CD95 gene. ${ }^{7}$ These findings indicate that the CD95-CD95 ligand system plays an important role in the maintenance of self-tolerance among both humans and mice. We now know that efficient apoptosis is a key element in maintaining peripheral lymphocyte homeostasis and in minimizing the accumulation of autoreactive lymphocytes. ${ }^{8}$ Important progress has been made during the past year in understanding the critical roles of a variety of molecules, especially a group of cysteine proteases, in the execution of apoptosis. ${ }^{2}$ These proteases appear to be the primary effector molecules responsible for apoptosis and their function may be critical for peripheral immunological tolerance. More recently, the functions of other apoptotic-related genes, such as Nurr77 ${ }^{9}$ and 'tissue' transglutaminase, ${ }^{10}$ have been linked to breakdown of tolerance and development of autoimmune diseases. In particular, the post-translational modification of glutamine and lysine residues of proteins catalyzed by 'tissue' transglutaminase might play an important role in apoptosis-related autoimmunity. ${ }^{10}$ Overexpression of tTG reduces the release of macromolecules (dsDNA and LDH) characterizing TNF $\alpha$-induced death of L929 cells. ${ }^{10}$ This suggests that tTG-catalyzed intracellular protein crosslinking plays an important role in stabilizing apoptotic bodies and preventing the leakage of their content. ${ }^{11}$ This phenomenon might represent a key feature of apoptosis in vivo, since a controlled disposal of cross-linked apoptotic bodies is necessary to prevent the inflammatory response as well as the exposure of autoantigens which may lead to the development of autoimmunity.

Although defects in the CD95/CD95L system that mediates apoptosis have been shown to be an important factor in autoimmune diabetes, thyroiditis and in the lymphoproliferation disorder described in MRL/Mp-/pr/lpr mice, ${ }^{12-14}$ the complex pathogenesis of these autoimmune diseases cannot be fully explained by an alteration of one signaling pathway alone. The dysfunction of other gene products, particularly of those involved in the execution/ disposal mechanisms of apoptosis, has also been hypothesized. A deregulated tTG is present in autoimmunity-prone MRL/pr/lpr mice. ${ }^{10}$ The reduced cross-linking activity of the enzyme is paralleled by the presence of autoantibodies against tTG and an abnormal accumulation of $\mathrm{LDH}$ and dsDNA in the blood. ${ }^{10}$

The presence of cryptic molecular determinants has been proposed to play a central role in the induction of the pathogenic autoimmune response. ${ }^{3}$ The question then arises as to how apoptosis results in the formation of harmful inciting neo-determinants. In addition to the autoantibodies against $\mathrm{tTG},{ }^{9,15}$ the presence of immunoglobulins (IgG) against its substrate proteins have been demonstrated in various autoimmune diseases. ${ }^{16}$ This phenomenon is particularly interesting considering that many tTG substrate proteins are also cleaved by caspases and calpains during apoptosis. It is tempting to hypothesize that the tTG-dependent polymerization of the polypeptides generated by proteolytic action during the execution phase of apoptosis represents a safety mechanism for the organism. Indeed, the accumulation of cleavage products, which are not present in viable cells, might be a harmful event contributing to the development of autoimmunity. In the absence of an active tTG, these polypeptides, which might represent a 'new-self', can be released into the extracellular space and/or presented as antigens, with the consequent generation of autoimmune responses. 


\section{References}

1. Theofilopoulos AN (1995) Immunol. Today 16: 150-159

2. Hale AJ, Smith CA, Sutherland LC, Stoneman VEA, Longthorne VL, Culhane AC and Williams GT (1996) Eur. J. Biochem. 236: 1-26

3. Ren Y and Savill J (1998) Cell Death Differ. 5: 563-568

4. Rosen A and Casciola-Rosen L (1999) Cell Death Differ. 6: 6-12

5. Ohsako S and Elkon KB (1999) Cell Death Differ. 6: 13-21

6. Nagata S and Golstein P (1995) Science 267: 1449-1456

7. Debatin KM (1996) Cell Death Differ. 3: 185-190

8. Peter ME, Hischkel FC, Hellbardt S, Chinnaiyan AM, Krammer PH and Dixit VM (1996) Cell Death Differ. 3: 161-170
9. Chan FK, Chen A and Winoto A (1998) J Immunol. 161: $4252-4256$

10. Piredda L, Amendola A, Colizzi V, Davies PJA, Farrace MG, Fraziano M, Gentile V, Uray I, Piacentini M and Fesus L (1997) Cell Death Differ. 4: 463-472

11. Piacentini M (1995) Curr Top. Microbiol. Immunol. 200: 163-176

12. Watanabe-Fukunaga $\mathrm{R}, \mathrm{Brannan} \mathrm{Cl}$, Copeland NG, Jenkins $\mathrm{NA}$ and Nagata $\mathrm{S}$ (1992) Nature 356: $314-317$

13. Benoist $C$ and Mathis D (1997) Cell 89: $1-3$

14. Giordano C, Papoff R, Ruberti G and Testi R (1997) Science 275: 960-963

15. Dieterich W, Ehnis T, Bauer M, Donner P, Volta U, Riecken E and Schuppan D (1997) Nature Med. 3: 797-801

16. Oliverio S, Amendola A, Di Sano F, Farrace MG, Fesus L, Nemes Z, Piredda L, Spinedi A and Piacentini M (1997) Mol. Cell. Biol. 17: 6040-6048 